\title{
The effect of probiotics on paediatric patients with inflammatory bowel disease: a systematic review
}

Inflammatory bowel diseases (IBD) can manifest at any age, but around a quarter of Crohn's Disease and Ulcerative Colitis patients present with symptoms before 20 years of age ${ }^{(1,2)}$. IBD is associated with significant psychosocial challenges causing patients to restrict aspects of their lifestyle and potentially impacting upon quality of life. The aetiology of IBD includes genes, the environment, and the microbiome. Recent studies in adults have shown that patients with IBD have lower microbial biodiversity, reduced abundance of beneficial bacteria such as Bifidobacterium and Lactobacillus and higher levels of pathogenic bacteria such as Clostridium difficile in comparison to healthy individuals ${ }^{(3)}$. Probiotics are defined as live microorganisms that confer a health benefit and therefore have potential as a therapy for $\mathrm{IBD}^{(4)}$. There is a significant amount of data that probiotics are beneficial in a variety of gastroenteric conditions but their effect on paediatric patients with IBD is uncertain.

A systematic review was conducted to assess the evidence for probiotics on paediatric patients with IBD, with outcomes assessed including induction or maintenance of remission, or changes in other biomarkers of disease status. MEDLINE, EMBASE, CINHAL, AMED, Cochrane and PROSPERO electronic databases were searched.

Six studies with sample sizes ranging from $n=4$ to $n=75$ met the criteria for inclusion in this review. The interventions included the probiotics Lactobacillus rhamnosus (LGG), Escherichia coli Nissle and a mixed strain probiotic preparation (VSL\#3). Probiotics were provided for intervention periods ranging from 10 days to 1 year. 4/6 studies demonstrated that probiotics induced or maintained remission in paediatric patients. Two studies also examined biomarkers of inflammation or disease status. This included a randomised double-blind trial which identified significantly improved clinical scores as assessed by colonoscopy and histology. No serious adverse events associated with probiotic intervention were reported in any of the studies included in this review. 4/6 studies within the review were open-label studies, and so were rated as having a high risk of bias using the Critical Appraisal Skills Programme Randomised controlled trial standard checklist.

There is initial evidence to support the statement that probiotics confer a therapeutic benefit to paediatric patients with IBD by inducing and maintaining remission. However, evidence for the underpinning mechanisms is limited due to the scarcity of doubleblind placebo controlled trials to date. Future research should further explore mechanisms by including microbiome and inflammatory biomarker analysis in their design, together with other important functional outcomes for paediatric IBD patients such as quality of life scores and measures of growth.

\section{References}

1. Shivananda S, Logan R, et al (1996) Gut 39, 690-697.

2. Kelsen J, Baldassano RN, et al. (2008) Inflamm Bowel Dis 14, S9-S11.

3. Franzosa EA, Sirota-Madi A, et al. (2019) Nat Microbiol 4, 293-305.

4. Hill C, et al. (2014) Nat Rev Gastroenterol \& Hepatol (2014) 11, 506-14. 